\title{
Sequential Adjuvant Chemotherapy and Radiotherapy in Treatment of Early Stage Endometrial Carcinoma: Single Institutional Experience
}

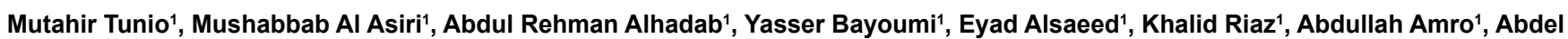
Salam Ismail ${ }^{2}$ and Abdel Aziz AlObaid ${ }^{3}$

${ }^{1}$ Radiation oncology, King Fahad Medical City (KFMC), Riyadh, Saudi Arabia

${ }^{2}$ Medical Oncology, King Fahad Medical City (KFMC), Riyadh, Saudi Arabia

${ }^{3}$ Gynecology Oncology, King Fahad Medical City (KFMC), Riyadh, Saudi Arabia

\begin{abstract}
Background: Aim was to evaluate the additional benefit of adjuvant chemotherapy in patients of early stage endometrial carcinoma $(E C)$ with adverse features.

Materials and methods: Between June 2006 and July 2011, 56 patients with EC after surgery were randomized to receive either adjuvant radiotherapy (RT) [35 patients] or adjuvant sequential chemotherapy and radiotherapy (CRT) [21 patients]. Median age was 57.6 years (40-80). Predominant stages were FIGO IB (44.6\%) and IIA (26.7\%). Mean body mass index was $35.9 \mathrm{~kg} / \mathrm{m}^{2}(23-72)$.

Results: Median follow-up was 55 months (6-60). The Kaplan-Meier estimates for loco regional control (LRC), distant metastasis control (DMC) and overall survival (OS) for RT and CRT arms were; $85.7 \%$ vs. $74.2 \%(p$ 0.04), $85.7 \%$ vs. $85.7 \%(p 0.9)$ and $82.8 \%$ vs. $81 \%(p 0.8)$ respectively. Patients in CRT arm had earlier and higher pelvic recurrences \{hazard ratios of 2.21 (1.45-7.85)\}. Acute hematological grade3 toxicity was higher in CRT arm (9.5\%) and no difference in acute or delayed non-hematological toxicities was seen between two arms.

Conclusion: Adjuvant chemotherapy in patients with EC after surgery is associated with inferior LRC and no additional benefit in DMC and OS. If adjuvant chemotherapy is considered it shall be given after adjuvant radiotherapy.
\end{abstract}

Keywords: Early stage; Endometrial carcinoma; Adjuvant radiotherapy; Adjuvant chemotherapy; Treatment outcomes

Abbreviations: EC: Endometrial Carcinoma; RT: Radiotherapy; CRT: Chemotherapy Radiotherapy; FIGO: International Federation of Gynecology and Obstetrics; LRC: Loco Regional Control; DMC: Distant Metastasis Control; PFS: Progression Free Survival; OS: Overall Survival; PORTEC: Post-Operative Radiation Therapy in Endometrial Cancer; GOG: Gynecologic Oncology Group; IRB: Institutional Ethical Review; CT: Computed Tomography; CTV: Clinical Target Volume; PTV: Planning Target Volume; 3DCRT: Three Dimensional Conformal Radiation Therapy; BMI: Body Mass Index

\section{Introduction}

Endometrial carcinoma (EC) is the tenth most common and the second most common gynecologic malignancy in women in the Saudi Arabia [1]. Surgery is the primary treatment involving a total abdominal hysterectomy, bilateral salpingo-oophorectomy, pelvic and para-aortic lymphadenectomy, and pelvic washings, with five year survival rates of $78 \%$ [2,3]. Randomized trials by Post-operative Radiation therapy in endometrial cancer (PORTEC) and Gynecological Oncology Group 99 (GOG-99) have shown significant reduction of the risk of pelvic and vaginal recurrence by adjuvant radiotherapy, although a survival benefit is not yet proven $[4,5]$. Thus radiotherapy remains mainstay of adjuvant treatment.

The role of chemotherapy alone in postoperative management of EC has remained controversial. Large randomized trial of Gynecologic Oncology Group (GOG 122) has shown the improvement in both progression free survival (PFS) and overall survival (OS) at 52 months with the use of adjuvant chemotherapy alone compared with adjuvant radiotherapy in stage III and IV patients (without evidence of hematogenous metastases) after surgery [6]. Contrary, two large randomized trials have shown that adjuvant chemotherapy alone was not better than adjuvant radiotherapy alone with no difference in PFS and OS rates in patients with early stage EC $[7,8]$.

Benefit of sequential adjuvant chemotherapy and radiotherapy was seen in advanced stage endometrial carcinoma by NSGO/EORTC and MaNGO studies with $36 \%$ reduction in the risk for relapse (hazard ratio (HR) $0.64,95 \%$ confidence interval (CI) $0.41-0.99$; $p$ 0.04) [9]. However, both trials of sequential adjuvant treatment failed to see any significant differences in the OS.

Theoretically, adjuvant chemotherapy after surgery may delay the curative radiotherapy which may result in locoregional control. The aim of our study was to evaluate impact of sequential chemotherapy and radiotherapy in patients with early stage endometrial carcinoma with adverse features.

\section{Materials and Methods}

After approval from Institutional Ethical Review Board (IRB) committee, patients referred to our department between June 2007 and July 2011 were selected when they met the following eligibility criteria:

*Corresponding author: Mutahir Tunio, MBBS, FCPS (Radiation Oncology), Assistant Consultant, Radiation Oncology, Comprehensive Cancer Center, King Fahad Medical City (KFMC), Riyadh 59046, Saudi Arabia, Tel: +966 1 2889999; Fax: 9661 4614006; E-mail: drmutahirtonio@hotmail.com

Received June 14, 2012; Accepted June 23, 2012; Published June 25, 2012

Citation: Tunio M, Asiri MA, Alhadab AR, Bayoumi Y, Alsaeed E, et al. (2012) Sequential Adjuvant Chemotherapy and Radiotherapy in Treatment of Early Stage Endometrial Carcinoma: Single Institutional Experience. J Nucl Med Radiat Ther S6:010. doi:10.4172/2155-9619.S6-010

Copyright: ( $) 2012$ Tunio M, et al. This is an open-access article distributed unde the terms of the Creative Commons Attribution License, which permits unrestricted use, distribution, and reproduction in any medium, provided the original author and source are credited. 
Citation: Tunio M, Asiri MA, Alhadab AR, Bayoumi Y, Alsaeed E, et al. (2012) Sequential Adjuvant Chemotherapy and Radiotherapy in Treatment of Early Stage Endometrial Carcinoma: Single Institutional Experience. J Nucl Med Radiat Ther S6:010. doi:10.4172/2155-9619.S6-010

Page 2 of 6

(1) histological confirmed endometrial carcinoma, (2) International Federation of Gynecology Obstetrics (FIGO) stage IA-IIB, (3) presence of adverse histological features (grade 3 , lymphovascular invasion), (4) Eastern Cooperative Oncology Group performance status 0-2; and (5) normal hematological parameters (hemoglobin $\geq 10 \mathrm{~g} / \mathrm{dL}$, white blood cell $\geq 4000 / \mathrm{mm}^{3}$, absolute neutrophil count $\geq 1500 / \mathrm{mm}^{3}$ and platelets $\geq 100000 / \mathrm{mm}^{3}$ ), normal hepatic parameters (serum bilirubin level $\leq$ $1.5 \mathrm{mg} / \mathrm{dL}$ and liver transaminase levels $\leq 3$ times upper normal limit) and normal renal function (serum creatinine level $\leq 1.5 \mathrm{mg} / \mathrm{dL}$ ).

Patients who had metastatic or recurrent disease or poor functional status were excluded.

After selection, patients were randomized to receive; Arm A: adjuvant pelvic radiotherapy and Arm B: adjuvant sequential chemotherapy followed by pelvic radiotherapy.

\section{Treatment protocol}

Radiotherapy: All patients were simulated using Siemens Emotions 6 computed tomography (CT) simulator. Contrast enhanced axial images of $5 \mathrm{~mm}$ slice thickness were obtained from the top of fourth lumbar (L4) vertebra to $5 \mathrm{~cm}$ below ischial tuberosities. After the acquisition of CT data, delineation of contouring of CTV [vaginal, cuff, parametria, external, internal iliac, presacral and common iliac lymph nodes], planning target volume $(\mathrm{PTV})=\mathrm{CTV}+1 \mathrm{~cm}$ margin, and critical structures (urinary bladder, rectum, small bowel) was performed using Varian Eclipse Contouring software by two radiation oncologists. After contouring treatment planning for conformal therapy (3DCRT) was carried out by two medical physicists. Treatment plans were made using box field technique for 3DCRT. The PTV was prescribed to 45-50.4 Gy in 25-28 fractions, 1.8 Gy per fraction, one fraction per day. Efforts were made to receive $45-50.4$ Gy to $95 \%$ of PTV and to reduce hot spots less than $120 \%$. During planning, total doses to the small bowel, rectum and bladder were constrained to $<40 \mathrm{~Gy}$, < $45 \mathrm{~Gy}$ and $45 \mathrm{~Gy}$ respectively. After completion of pelvic external beam irradiation, high dose rate (HDR) intravaginal brachytherapy (IVBT) was given. Total IVBT dose was 15 Gy delivered in 3 sessions (each session three days apart). The reference point for dose prescription was $0.5 \mathrm{~cm}$ from surface of vaginal applicators figure 1 .

Chemotherapy: In arm B, adjuvant chemotherapy was given initially 6 weeks after surgery, before starting radiation therapy. Adjuvant chemotherapy consisted of four cycles of paclitaxel $(175 \mathrm{mg} /$ $\left.\mathrm{m}^{2}\right)$ and carboplatin $\left(350 \mathrm{mg} / \mathrm{m}^{2}\right)$ every 21 days based on Lupe $\mathrm{K}$, et al. Protocol [10]. Dose modifications were done according to side effects and tolerance of patients.

Toxicity and Response evaluation: During radiation therapy, patients were evaluated every week for weight, performance status, hematology/chemistry and side effects. The National Cancer Institute Common Toxicity Criteria (NCI-CTC) version 2.0, were used to score acute radiation toxicity ( $\leq 90$ days from start of radiation therapy). The Radiation Therapy Oncology Group (RTOG) Late Radiation Morbidity Scoring Criteria were used to score radiation toxicity persisting beyond 90 days from the completion of radiotherapy.

After completion of therapy, periodic follow ups were carried out every 3 months for first two years and every 6 months subsequent $3^{\text {rd }}$ to $5^{\text {th }}$ year with physical and per vaginal examination, pap smear and CT chest, abdomen and pelvis.

Statistical analysis: The primary endpoints were locoregional and distant control, PFS and OS. Secondary endpoints were toxicity profile. The times to last follow up evaluation, appearance of local and distant relapse and death were calculated from date of starting treatment. DFS was defined as the duration between the entry date and the date of documented disease reappearance, death from cancer and/or last follow-up (censored). OS was defined as the duration between the entry date and the date of patient death or last follow-up (censored). Probabilities of locoregional/distant control, PFS and OS were determined with the Kaplan-Meier method. The comparisons for various endpoints were performed using log rank test and Cox regression analysis. Univariate and multivariate analyses were also performed for different prognostic factors. All statistical analyses were performed using the computer program SPSS version 16.0.

\section{Results}

Median follow up was 55 months (range: 6-60 months). There was no significant difference in patients' characteristics in both treatment subgroups table 1 . Majority of study cohort was with cormorbids (29 patients, $51.8 \%$ ) and median BMI was $35.9 \mathrm{~kg} / \mathrm{m}^{2}$ (range: $28-72$ ). Most common stage was, IB in 25 patients $(44.6 \%)$ followed by IIA in 15 patients (26.7\%) and node positive (N1) disease in 4 patients (7.1\%).

Median time between surgery and radiotherapy in arm A was 7.1 weeks (range: $5-24)$ and in arm B was 13 weeks (11-18) p value 0.02 . The median dose to PTV was $47.5 \mathrm{~Gy}$ (range: $45-50.4$ )in both arms and IVBT dose was $15 \mathrm{~Gy}$ at $0.5 \mathrm{~cm}$ from surface of applicators in both arms and mean radiotherapy duration was 6.5 weeks (range: 6- 8).

\section{Toxicity profile}

Radiotherapy treatment was generally well tolerated by all patients in both arms with grade 1 and 2 acute side effects table 2 . However, in chemotherapy/radiotherapy arm, two patients developed febrile Neutropenia (9.5\%) during chemotherapy, which required hospitalization and Granulocyte- colony stimulating factor (GCSF) support. Late toxicity was seen only in one patient in radiotherapy arm, who presented with sub-acute intestinal obstruction which was managed conservatively.

\section{Locoregional control, distant control and overall survival rates}

The Kaplan-Meier estimates of locoregional control, distant

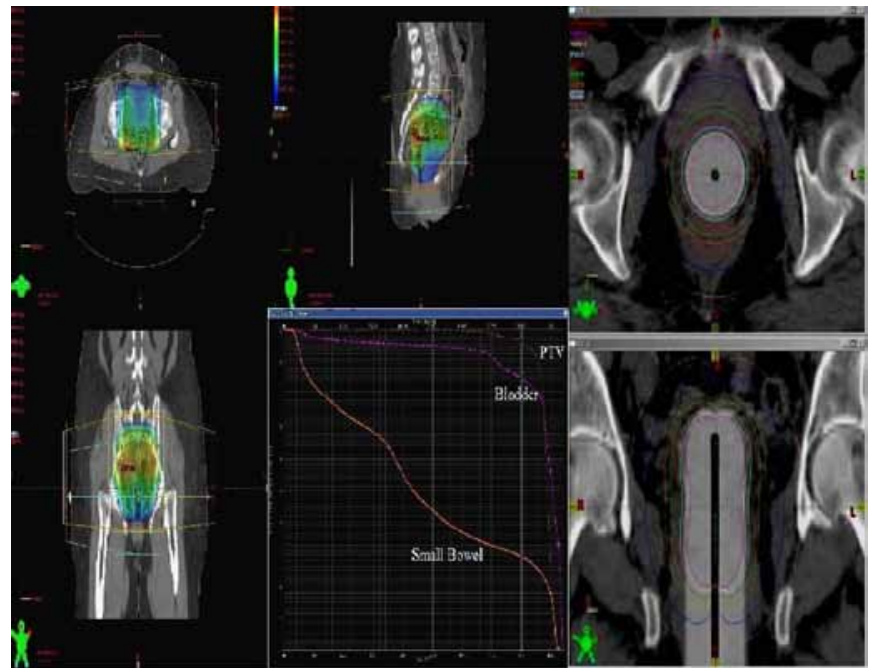

Figure 1: Pelvic box field technique using three dimensional conformal external radiation therapy plan delivering $45 \mathrm{~Gy}$ in 25 fractions followed by vaginal high dose rate brachytherapy by using vaginal single channel applicator. 
Citation: Tunio M, Asiri MA, Alhadab AR, Bayoumi Y, Alsaeed E, et al. (2012) Sequential Adjuvant Chemotherapy and Radiotherapy in Treatment of Early Stage Endometrial Carcinoma: Single Institutional Experience. J Nucl Med Radiat Ther S6:010. doi:10.4172/2155-9619.S6-010

Page 3 of 6

\begin{tabular}{|c|c|c|c|}
\hline Variables & $\begin{array}{l}\text { Radiotherapy } \\
\text { Alone } \\
\mathrm{N}=35(62.5 \%)\end{array}$ & $\begin{array}{l}\text { Sequential Chemotherapy and radiotherapy } \\
\mathrm{N}=21(37.5 \%)\end{array}$ & $P$ value \\
\hline $\begin{array}{l}\text { Comorbids } \\
\text { (DM/HTN) } \\
\text { Yes } \\
\text { No }\end{array}$ & $\begin{array}{l}18(51.5 \%) \\
17(48.5 \%)\end{array}$ & $\begin{array}{l}11(52.4 \%) \\
10(47.6 \%)\end{array}$ & 0.9 \\
\hline $\begin{array}{l}\text { FIGO Stage } \\
\text { IA } \\
\text { IB } \\
\text { IIA } \\
\text { IIB }\end{array}$ & $\begin{array}{l}2(5.7 \%) \\
16(45.7 \%) \\
9(25.8 \%) \\
8(22.8 \%)\end{array}$ & $\begin{array}{l}- \\
9(43.0 \%) \\
6(28.5 \%) \\
6(28.5 \%)\end{array}$ & 0.6 \\
\hline $\begin{array}{l}\text { Cell Type } \\
\text { Endometroid } \\
\text { Serous papillary } \\
\text { Clear cell }\end{array}$ & $\begin{array}{l}33(94.2 \%) \\
1(2.9 \%) \\
1(2.9 \%)\end{array}$ & $\begin{array}{l}19(90.5 \%) \\
2(9.50 \%) \\
-\end{array}$ & 0.8 \\
\hline $\begin{array}{l}\text { Pathological grade } \\
\text { G1 } \\
\text { G2 } \\
\text { G3 }\end{array}$ & $\begin{array}{l}5(14.2 \%) \\
12(34.3 \%) \\
18(51.5 \%)\end{array}$ & $\begin{array}{l}4(19.0 \%) \\
8(38.0 \%) \\
9(43.0 \%)\end{array}$ & 0.9 \\
\hline $\begin{array}{l}\text { LVI } \\
\text { Yes } \\
\text { No }\end{array}$ & $\begin{array}{l}12(34.3 \%) \\
23(65.7 \%)\end{array}$ & $\begin{array}{l}6(28.5 \%) \\
15(71.5 \%)\end{array}$ & 0.7 \\
\hline $\begin{array}{l}\text { ER/ PR Receptors } \\
\text { Positive } \\
\text { Negative } \\
\text { unknown }\end{array}$ & $\begin{array}{l}6(17.1 \%) \\
7(20.0 \% \%) \\
22(62.9 \%)\end{array}$ & $\begin{array}{l}2(9.5 \%) \\
4(19.0 \%) \\
15(71.5 \%)\end{array}$ & 0.8 \\
\hline $\begin{array}{l}\text { LN } \\
\text { Positive } \\
\text { Negative }\end{array}$ & $\begin{array}{l}3(8.6 \%) \\
32(91.4 \%)\end{array}$ & $\begin{array}{l}1(4.8 \%) \\
20(95.2 \%)\end{array}$ & 0.8 \\
\hline $\begin{array}{l}\text { Squamous Metaplasia } \\
\text { Yes } \\
\text { No }\end{array}$ & $\begin{array}{l}3(8.6 \%) \\
32(91.4 \%)\end{array}$ & $\begin{array}{l}1(4.8 \%) \\
20(95.2 \%)\end{array}$ & 0.9 \\
\hline $\begin{array}{l}\text { Dose RT } \\
45 \text { Gy EBRT + VBT } 15 \text { Gy } \\
50.4 \text { Gy EBRT+ VBT } 15 \text { Gy }\end{array}$ & $\begin{array}{l}23(65.7 \%) \\
12(34.3 \%)\end{array}$ & $\begin{array}{l}11(52.4 \%) \\
10(47.6 \%)\end{array}$ & 0.8 \\
\hline
\end{tabular}

$\mathrm{DM}=$ diabetes, $\mathrm{HTN}=$ hypertension, $\mathrm{FIGO}=$ International Federation of Gynecology and Obstetrics, G= grade, LVI= lymphovascular invasion, LN= Iymph nodes, EBRT= external beam radiation therapy, $\mathrm{VBT}=$ vaginal brachytherapy, $\mathrm{RT}=$ radiation therapy, $\mathrm{ER}=$ estrogen receptors, $\mathrm{PR}=$ progesterone receptors

Table 1: Patients characteristics according to adjuvant treatment category.

\begin{tabular}{|c|c|c|c|c|c|c|c|c|c|c|c|c|}
\hline \multirow[t]{2}{*}{ Treatment subgroups } & \multicolumn{2}{|c|}{ Hematological } & \multicolumn{2}{|l|}{ Skin } & \multicolumn{2}{|c|}{ Small Bowel } & \multicolumn{2}{|l|}{ Proctitis } & \multicolumn{2}{|l|}{ Cystitis } & \multicolumn{2}{|c|}{ Vagintis (discharge, pain) } \\
\hline & $\mathbf{G}<3$ & G>3 & $\mathbf{G}<3$ & G >3 & $\mathbf{G}<3$ & $\mathrm{G}>3$ & $\mathbf{G}<3$ & $\mathbf{G}>\mathbf{3}$ & $\mathbf{G}<3 \quad \mathbf{G}$ & $G>3$ & $\mathbf{G}<3$ & $\mathbf{G}>3$ \\
\hline $\begin{array}{l}\text { Arm A: Adjuvant radiotherapy } \\
\text { alone } N=35\end{array}$ & $\begin{array}{ll}\mathrm{A} & 1 \\
\mathrm{~N} & 0 \\
\mathrm{~T} & 0\end{array}$ & $\begin{array}{l}0 \\
0 \\
0\end{array}$ & $\begin{array}{l}\text { DD } 2(5.7 \%) \\
\text { WD } 3(8.5 \%)\end{array}$ & $\begin{array}{l}0 \\
0\end{array}$ & $\begin{array}{l}\text { NV } 5(14.3 \%) \\
\text { AC } 3(8.5 \%)\end{array}$ & $\begin{array}{l}0 \\
0\end{array}$ & $2(5.7 \%)$ & 0 & $1(2.8 \%)$ & 0 & $2(5.7 \%)$ & 0 \\
\hline $\begin{array}{l}\text { Arm } B \text { : Adjuvant chemotherapy } \\
\text { and radiotherapy } N=21\end{array}$ & $\begin{array}{ll}\text { A } & 2(9.5 \%) \\
\text { N } & 3(14.2 \%) \\
\text { T } & 4(19.0 \%)\end{array}$ & $\begin{array}{c}0 \\
2(9.5 \%) \\
0\end{array}$ & $\begin{array}{l}\text { DD } 1(4.7 \%) \\
\text { WD } 2(9.5 \%)\end{array}$ & $\begin{array}{l}0 \\
0\end{array}$ & $\begin{array}{l}\text { NV } 3(14.2 \%) \\
\text { AC } 2(9.5 \%)\end{array}$ & $\begin{array}{l}0 \\
0\end{array}$ & $2(9.5 \%)$ & 0 & $1(4.7 \%)$ & 0 & $2(9.5 \%)$ & 0 \\
\hline
\end{tabular}

$A=$ Anemia,$N=$ Neutropenia,$T=$ thrombocytopenia, $D D=$ dry desquamation, $W D=$ wet desquamation, $N V=$ nausea and vomiting, $A P=$ abdominal cramps

Table 2: Acute Toxicity Profile in both treatment subgroups.

\begin{tabular}{|c|c|c|c|}
\hline Recurrence & Time of first recurrence & Salvage Treatment & Status At 55 months \\
\hline $\begin{array}{l}\text { Locoregional } \\
9 \text { patients } \\
\text { Vaginal } \\
2(3.02 \%)\end{array}$ & $\begin{array}{l}23 \text { months } \\
10 \text { months }\end{array}$ & $\begin{array}{l}\text { Surgery } \\
\text { Surgery }\end{array}$ & $\begin{array}{l}1 \text { dead with disease } \\
1 \text { disease free alive }\end{array}$ \\
\hline $\begin{array}{l}\text { Pelvic } \\
7(12.5 \%)\end{array}$ & $\begin{array}{l}38 \text { months } \\
30 \text { months } \\
23 \text { months }\end{array}$ & Chemotherapy & $\begin{array}{l}6 \text { dead with disease } \\
1 \text { disease free alive }\end{array}$ \\
\hline $\begin{array}{l}\text { Distant } 6 \text { patients } \\
\text { Lungs } \\
3(5.3 \%) \text { Bones } \\
1(1.8 \%) \text { Para-aortic nodes } \\
2(3.5 \%)\end{array}$ & $\begin{array}{l}23 \text { months } \\
31 \text { months } \\
23 \text { months }\end{array}$ & $\begin{array}{l}\text { Chemotherapy } \\
\text { Chemotherapy and bisphosphonates } \\
\text { Chemotherapy }\end{array}$ & $\begin{array}{l}3 \text { dead with disease } \\
1 \text { disease free alive } \\
1 \text { disease free alive } \\
1 \text { dead with disease }\end{array}$ \\
\hline
\end{tabular}

Table 3: Recurrence Pattern in patients in both treatment subgroups. 
Citation: Tunio M, Asiri MA, Alhadab AR, Bayoumi Y, Alsaeed E, et al. (2012) Sequential Adjuvant Chemotherapy and Radiotherapy in Treatment of Early Stage Endometrial Carcinoma: Single Institutional Experience. J Nucl Med Radiat Ther S6:010. doi:10.4172/2155-9619.S6-010

Page 4 of 6

metastasis control, and overall survival were $80.1 \%, 85.5 \%, 78.6 \%$ and $81.9 \%$ respectively figure 2 .

Nine patients developed locoregional recurrences (in-field). Two patients had vaginal recurrences $(3.02 \%)$ and 7 patients had pelvic nodal recurrence (12.5\%). Higher and earlier locoregional recurrences ( 5 patients, $8.9 \%$ ) were seen in adjuvant chemotherapy and radiotherapy arm ( $\mathrm{p}$ value 0.04 ). For all vaginal failures, salvage surgery was done and for pelvic nodal failures salvage chemotherapy was given in 5 patients $(8.9 \%)$ table 3 .

Distant metastases were seen 6 patients (10.7\%) of whom 3 patients (5.3\%) had simultaneous locoregional failures. First event was seen at
23 months of completion of treatment. All patients received salvage chemotherapy. No significant difference in distant control was seen between two arms. At 55 months of follow-up, 10 patients (17.8\%) were dead. Of whom 6 patients (10.7\%) died of non endometrial cancer related causes. No difference in DFS and OS was found between two treatment arms.

Further univariate and multivariate analyses were carried out table 4. Chemotherapy, BMI $>30 \mathrm{Kg} / \mathrm{m}^{2}$ and LVI were found poor prognostic factors for locoregional failure ( $p 0.03,0.02$ and 0.03 respectively). For distant failure, FIGO stage was poor prognostic factor $(p 0.02)$ and for OS, BMI and FIGO stage were poor prognostic factors ( $p 0.01$ and 0.02 respectively).

\begin{tabular}{|c|c|c|c|}
\hline Variable & $\begin{array}{l}\text { Locoregional Control } \\
\mathrm{P} \text { value OR }(95 \% \mathrm{CI})\end{array}$ & $\begin{array}{l}\text { Distant Metastasis Control } \\
P \text { value OR }(95 \% \mathrm{CI})\end{array}$ & $\begin{array}{l}\text { Overall Survival } \\
P \text { value OR }(95 \% \mathrm{CI})\end{array}$ \\
\hline Age (< 50 vs. $>50$ years) & $0.330 .93(0.90-1.50)$ & $0.661 .10(0.89-2.00)$ & $0.710 .50(0.10-2.41)$ \\
\hline Cormorbids (Yes vs. No) & $0.980 .88(0.67-0.97)$ & $0.901 .80 \quad(0.79-2.10)$ & $1.001 .80(0.79-2.10)$ \\
\hline FIGO stage $(<\mid B$ vs. $>$ IB) & $0.750 .50 \quad(0.10-2.41)$ & $0.023 .65(1.81-9.65)$ & $0.013 .85 \quad(1.91-10.35)$ \\
\hline N stage (N0 vs. N1) & $0.771 .10 \quad(0.89-2.00)$ & $0.661 .10(0.89-2.00)$ & $0.561 .21(1.10-2.10)$ \\
\hline BMI kg/m² (>30 vs. <30) & $0.023 .45 \quad(1.61-9.45)$ & $0.561 .10(0.89-2.00)$ & $0.023 .65(1.81-9.65)$ \\
\hline Cell type ( Endometroid vs. non Endometroid) & $0.481 .21 \quad(1.10-2.10)$ & $0.601 .10(0.89-2.00)$ & $0.771 .21 \quad(1.10-2.10)$ \\
\hline Grade (<G2 vs..G3) & $0.230 .97 \quad(0.95-1.13)$ & $0.900 .88(0.67-0.97)$ & $0.230 .97 \quad(0.95-1.13)$ \\
\hline LVI ( no vs. yes) & $0.053 .34 \quad(2.52-10.34)$ & $0.900 .88(0.67-0.97)$ & $0.601 .10(0.89-2.00)$ \\
\hline Adjuvant chemotherapy (No vs. Yes) & $0.03 \quad 2.21(1.45-7.85)$ & $0.440 .93(0.90-1.50)$ & $0.700 .50(0.10-2.41$ \\
\hline EBRT dose (45 Gy vs. 50.4) & $0.45 \quad 0.78 \quad(0.23-2.38)$ & $0.450 .78 \quad(0.23-2.38)$ & $0.561 .10(0.89-2.00)$ \\
\hline Squamous metaplasia (Yes vs. No) & $0.851 .80 \quad(0.79-2.10)$ & $0.400 .78 \quad(0.23-2.38)$ & $0.330 .93(0.90-1.50)$ \\
\hline
\end{tabular}

FIGO= International Federation of Gynecology and Obstetrics, BMI= body mass index, LVI= lymphovascular invasion, EBRT= external beam radiation therapy, G= grade Table 4: Univariate and multivariate analyses of variables on locoregional control, distant control and overall survival in both treatment sub groups.

\begin{tabular}{|c|c|c|c|c|c|c|c|c|c|}
\hline Study [references] & Stage & $\begin{array}{l}\text { Radiation } \\
\text { techniques }\end{array}$ & Chemotherapy & $\begin{array}{l}\text { Sample } \\
\text { size } \\
(n)\end{array}$ & $\begin{array}{l}\text { Follow up } \\
\text { period } \\
\text { (months) }\end{array}$ & $\begin{array}{l}\text { Disease } \\
\text { Free } \\
\text { Survival }\end{array}$ & $\begin{array}{l}\text { Overall } \\
\text { survival }\end{array}$ & $\begin{array}{l}\text { Grade } 3 \text { Acute } \\
\text { toxicity } \\
\text { incidence }\end{array}$ & $\begin{array}{l}\text { Grade } \\
3 \text { Late } \\
\text { toxicity }\end{array}$ \\
\hline $\begin{array}{l}\text { Lupe K, et al. } \\
\text { [10] }\end{array}$ & $\begin{array}{l}\text { FIGO } \\
\text { IIIA-IVA }\end{array}$ & $\begin{array}{l}\text { Pelvic RT } 45 \text { Gy } \pm \\
\text { para-aortic RT and } \\
\text { VBT }\end{array}$ & $\begin{array}{l}\text { Adjuvant Paclitaxel+ carboplatin } \times 4 \\
\text { before RT }\end{array}$ & 63 & 24 & $55 \%$ & $55 \%$ & $12 \%$ & $18 \%$ \\
\hline $\begin{array}{l}\text { Alvarez Secord } \\
\text { A, et al. [11] }\end{array}$ & $\begin{array}{l}\text { Retrospective } \\
\text { FIGO } \\
\text { IIIA- IVA }\end{array}$ & $\begin{array}{l}\text { Pelvic RT } 45 \text { Gy } \pm \\
\text { para-aortic RT and } \\
\text { VBT }\end{array}$ & NA & 83 & 36 & $62 \%$ & $79 \%$ & NA & NA \\
\hline $\begin{array}{l}\text { Geller MA, et al. } \\
\text { [12] }\end{array}$ & FIGO III-IVA & $\begin{array}{l}\text { Pelvic RT } 45 \text { Gy } \\
\pm \text { VBT }\end{array}$ & $\begin{array}{l}\text { Docetaxel+ carboplatin } \mathrm{C} 4 \rightarrow \mathrm{RT} \rightarrow \\
\text { Docetaxel+ carboplatin } \times \mathrm{C} 2\end{array}$ & 42 & 28 & $71 \%$ & $90 \%$ & $54 \%$ & NA \\
\hline $\begin{array}{l}\text { Secord AA, et } \\
\text { al. [13] }\end{array}$ & FIGO III-IVA & $\begin{array}{l}\text { Pelvic RT } 45 \text { Gy } \\
\pm \text { VBT }\end{array}$ & $\begin{array}{l}\text { Three arms: } \\
1 . \\
\text { C } \rightarrow \text { RT } \rightarrow \text { C } \\
\text { RT } \rightarrow \text { C } \\
2 . \\
\text { C } \rightarrow \text { RT }\end{array}$ & $\begin{array}{l}1 . \\
5 \\
2 . \\
8 \\
3 . \\
6\end{array}$ & 36 & \begin{tabular}{|l}
1. \\
$9 \%$ \\
2. \\
$7 \%$ \\
3. \\
$2 \%$
\end{tabular} & $\begin{array}{l}1 . \\
8 \% \\
2 . \\
4 \% \\
3 . \\
7 \%\end{array}$ & NA & NA \\
\hline $\begin{array}{l}\text { Fields AL, et al. } \\
\text { [14] }\end{array}$ & $\begin{array}{l}\text { Papillary } \\
\text { serous } \\
\text { carcinoma }\end{array}$ & $\begin{array}{l}\text { Pelvic RT } 45 \text { Gy } \pm \\
\text { para-aortic RT }\end{array}$ & $\begin{array}{l}\text { Adjuvant Paclitaxel+ carboplatin x C4 } \\
\text { before RT }\end{array}$ & 30 & 36 & $54-69 \%$ & $52-75 \%$ & $43 \%$ & NA \\
\hline Present study & FIGO IA-IIB & $\begin{array}{l}\text { Pelvic RT } 45 \text { Gy } \\
\text { +VBT } 15 \text { Gy }\end{array}$ & $\begin{array}{l}\text { Two arms } \\
1 . \\
\text { RT alone } \\
2 . \\
\text { Adjuvant Paclitaxel+ } \\
\quad \text { Carboplatin } x \\
\quad \text { C4 before RT }\end{array}$ & 56 & 55 & $78.6 \%$ & $81.9 \%$ & $9.5 \%$ & $1.7 \%$ \\
\hline
\end{tabular}

FIGO= International Federation of Gynecology and Obstetrics, $\mathrm{RT}=$ radiation therapy, VBT= vaginal brachytherapy, NA= not available, $\mathrm{xC}=$ cycles, $\mathrm{C}=$ chemotherapy

Table 5: Comparison of various studies using sequential chemotherapy and radiotherapy and its impact on treatment outcomes. 
Citation: Tunio M, Asiri MA, Alhadab AR, Bayoumi Y, Alsaeed E, et al. (2012) Sequential Adjuvant Chemotherapy and Radiotherapy in Treatment of Early Stage Endometrial Carcinoma: Single Institutional Experience. J Nucl Med Radiat Ther S6:010. doi:10.4172/2155-9619.S6-010
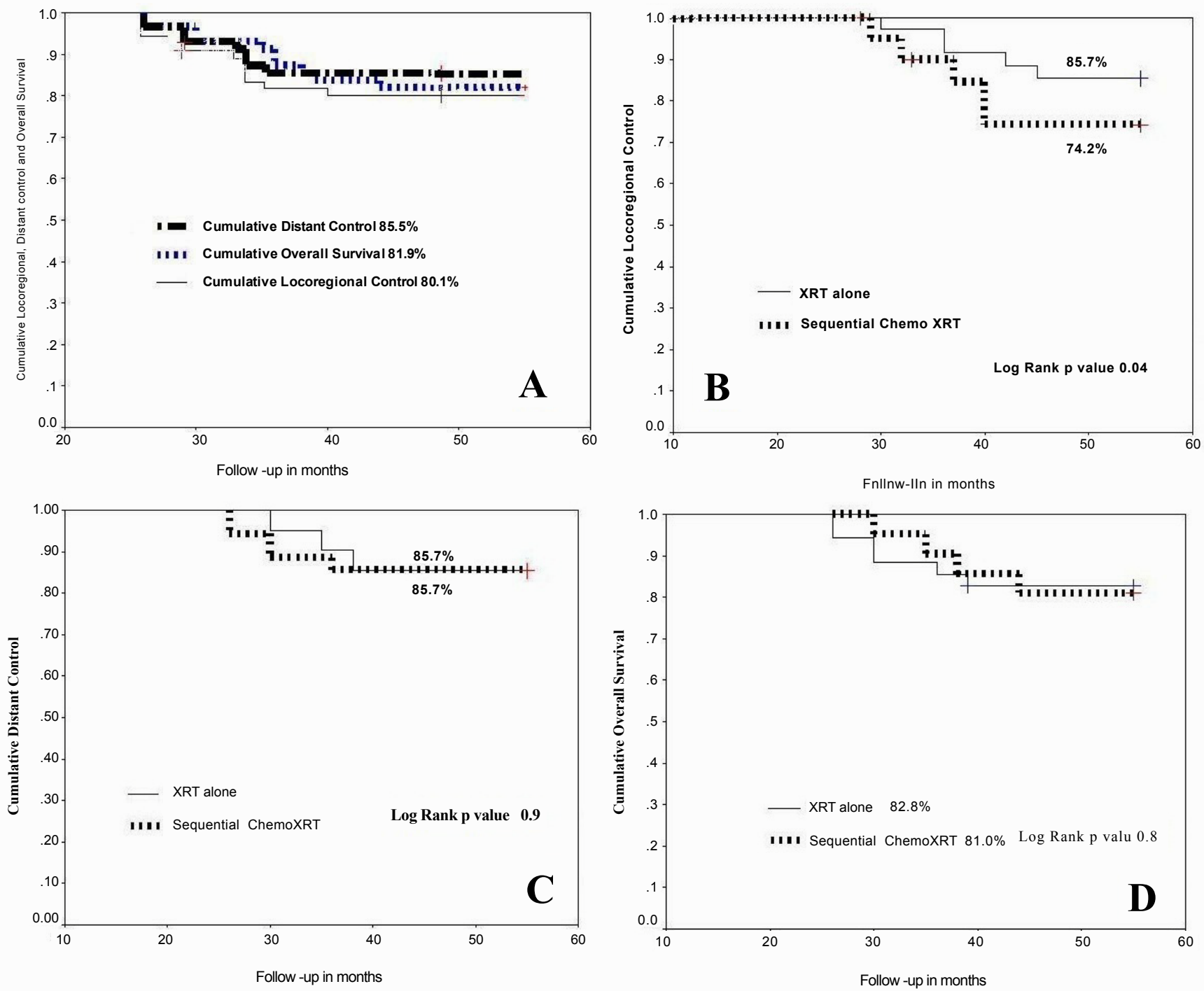

Figure 2: (a) cumulative locoregional control, distant control and overall survival rates, (b) locoregional control, (c) and (d) according to adjuvant radiotherapy and sequential chemotherapy/radiotherapy treatment subgroups.

\section{Discussion}

Adjuvant sequential chemotherapy and radiotherapy has been employed in FIGO stages III and IVA endometrial carcinoma after surgery, with minimal improvement in DFS and high hematological toxicity table 5. Our study is the first, which incorporated adjuvant sequential chemotherapy and radiotherapy in early stage (FIGO, I-IIB) endometrial carcinoma with adverse features.

The results of our study showed that adjuvant sequential chemotherapy and radiotherapy is associated with reduced locoregional control ( $p$ value 0.003 ) in patients with early stage endometrial carcinoma, yet there was no impact of additional adjuvant chemotherapy on distant control, DFS and OS. However sequential regimen was generally well tolerated, with grade 3 hematological toxicity encountered only in $9.5 \%$ of patients.

The possible explanation for high locoregional recurrences in adjuvant sequential chemotherapy and radiotherapy patients can be explained by delayed pelvic radiotherapy, i.e. mean time between surgery and radiotherapy was 13 weeks (11-18). However, previously published studies of adjuvant sequential regimen in advanced stage endometrial carcinoma remained fail to document poor locoregional control because of shorter follow up period (36 months) $[10,11,12,13]$.

No improvement in distant control and DFS and decreased toxicity in patients who received adjuvant sequential chemotherapy and radiotherapy can be explained by high body mass index (mean, $35.9 \mathrm{~kg} / \mathrm{m}^{2}$ (range: $28-72$ ) in our study cohort, which perhaps caused dose capping and subsequently led to suboptimal chemotherapy dose [15]. Presence of co-morbidities (51.8\%) and high body mass index in our cohort was associated with decreased OS, as also reported by other studies [16].

Potential strength to support our data was reasonable follow up period. Our study can be criticized for its low sample size and presence of confounders (obese patients with co-morbidities). 
Citation: Tunio M, Asiri MA, Alhadab AR, Bayoumi Y, Alsaeed E, et al. (2012) Sequential Adjuvant Chemotherapy and Radiotherapy in Treatment of Early Stage Endometrial Carcinoma: Single Institutional Experience. J Nucl Med Radiat Ther S6:010. doi:10.4172/2155-9619.S6-010

Page 6 of 6

\section{Conclusion}

Our results did not support the incorporation of sequential chemotherapy in adjuvant care of early endometrial carcinoma. However future large trials should consider the appropriate sequencing of chemotherapy, i.e. Adjuvant chemotherapy followed by radiotherapy or adjuvant radiotherapy followed by chemotherapy or sandwich radiotherapy between chemotherapy in early stage endometrial carcinoma with adverse features.

\section{References}

1. Cancer incidence report Saudi Arabia 1999-2000.

2. Berrino F, De Angelis R, Sant M, Rosso S, Bielska-Lasota M et al. (2007) Survival for eight major cancers and all cancers combined for European adults diagnosed in 1995-99: results of the EUROCARE-4 study. Lancet Oncol 8: 773-783.

3. Obermair A, Manolitsas TP, Leung Y, Hammond IG, McCartney AJ (2004) Total laparoscopic hysterectomy for endometrial cancer: patterns of recurrence and survival. Gynecol Oncol 92: 789-793.

4. Creutzberg CL, Nout RA, Lybeert ML, Wárlám-Rodenhuis CC, Jobsen JJ, et al (2011) Fifteen-year radiotherapy outcomes of the randomized PORTEC-1 trial for endometrial carcinoma. Int J Radiat Oncol Biol Phys 81: e631-638.

5. Keys HM, Roberts JA, Brunetto VL, Zaino RJ, Spirtos NM, et al. (2004) A phase III trial of surgery with or without adjunctive external pelvic radiation therapy in intermediate risk endometrial adenocarcinoma: a Gynecologic Oncology Group Study. Gynecol Oncol 92: 744-751.

6. Randall ME, Spirtos NM, Dvoretsky P (2003) Whole abdominal radiotherapy versus combination chemotherapy with doxorubicin and cisplatin in advanced endometrial carcinoma (phase III): Gynecologic Oncology Group Study No. 122. J Natl Cancer Inst Monogr 19: 13-15.

7. Maggi R, Lissoni A, Spina F, Melpignano M, Zola P, et al. (2006) Adjuvan chemotherapy vs. radiotherapy in high-risk endometrial carcinoma: results of a randomised trial. $\mathrm{Br} \mathrm{J}$ Cancer 95: 266-271.
8. Susumu N, Sagae S, Udagawa Y, Niwa K, Kuramoto H, et al. (2008) Randomized phase III trial of pelvic radiotherapy versus cisplatin-based combined chemotherapy in patients with intermediate and high-risk endometrial cancer: a Japanese Gynecologic Oncology Group study. Gynecol Oncol 108: 226-233.

9. Hogberg T, Signorelli M, de Oliveira CF, Fossati R, Lissoni AA, et al. (2010) Sequential adjuvant chemotherapy and radiotherapy in endometrial cancerresults from two randomised studies. Eur J Cancer 46: 2422-2431.

10. Lupe K, Kwon J, D'Souza D, Gawlik C, Stitt L, et al. (2007) Adjuvant paclitaxe and carboplatin chemotherapy with involved field radiation in advanced endometrial cancer: a sequential approach. Int J Radiat Oncol Biol Phys 67 $110-116$.

11. Alvarez Secord A, Havrilesky LJ, Bae-Jump V, Chin J, Calingaert B, et al (2007) The role of multi-modality adjuvant chemotherapy and radiation in women with advanced stage endometrial cancer. Gynecol Oncol 107: 285-291.

12. Geller MA, Ivy JJ, Ghebre R, Downs LS Jr, Judson PL, et al. (2011) A phase II trial of carboplatin and Docetaxel followed by radiotherapy given in a "sandwich" method for stage III, IV and recurrent endometrial cancer. Gynecol Oncol 121 112-117.

13. Secord AA, Havrilesky LJ, O'Malley DM, Bae-Jump V, Fleming ND, et al. (2009) A multicenter evaluation of sequential multimodality therapy and clinical outcome for the treatment of advanced endometrial cancer. Gynecol Oncol 114: $442-447$.

14. Fields AL, Einstein MH, Novetsky AP, Gebb J, Goldberg GL (2008) Pilot phase II trial of radiation "sandwiched" between combination paclitaxel/platinum chemotherapy in patients with uterine papillary serous carcinoma (UPSC) Gynecol Oncol 108: 201-206

15. Hunter RJ, Navo MA, Thaker PH, Bodurka DC, Wolf JK (2009) Dosing chemotherapy in obese patients: actual versus assigned body surface area (BSA). Cancer Treat Rev 35: 69-78.

16. Chia VM, Newcomb PA, Trentham-Dietz A, Hampton JM (2007) Obesity, diabetes and other factors in relation to survival after endometrial cancer diagnosis. Int J Gynecol Cancer 17: 441-446.

\footnotetext{
This article was originally published in a special issue, Cancer Radiation Therapy handled by Editor(s). Dr. XinChen, University of Arkansas for Medical Sciences, USA
} 